\title{
Tempo de Voo da Onda Difratada (ToFD) Auxilia na Garantia da Qualidade da Produção de Peças e Estruturas Mecânicas
}

\author{
R C Mayworm ${ }^{1}$ (D), A V Alvarenga ${ }^{1}$ (D), R P B Costa-Félix ${ }^{1}$ (D) \\ ${ }^{1}$ Instituto Nacional de Metrologia, Qualidade e Tecnologia - INMETRO, Laboratório de Ultrassom, Duque de Caxias, RJ, Brasil.
}

Como citar: Mayworm RC, Alvarenga AV, Costa-Félix RPB. Tempo de Voo da Onda Difratada (ToFD) auxilia na garantia da qualidade da produção de peças e estruturas mecânicas. Soldagem \& Inspeção. 2019;24:e2427. https://doi.org/10.1590/0104-9224/SI24.27

\begin{abstract}
Resumo: A Técnica Tempo de Voo da Onda Difratada (ToFD) é uma técnica de ensaio não destrutivo por ultrassom utilizada para a deteç̧ão de falhas e descontinuidades em diferentes componentes, garantindo a qualidade, confiabilidade e segurança. É amplamente utilizada na indústria, principalmente na avaliação de peças e estruturas mecânicas. É largamente utilizada nos setores petróleo/petroquímico, químico, aeronáutico, aeroespacial, siderúrgico, naval, eletromecânico, papel e celulose, entre outros. Seu desenvolvimento e implementação no Laboratório de Ultrassom do Inmetro foi baseado na norma ISO 16828:2012 (Non-Destructive testing - Ultrasonic Testing - Time-ofFlight Diffraction Technique as a Method for Detection and Sizing of Discontinuities). Tendo controle sobre as diferentes características do sistema, desde a especificação das suas características ultrassônicas, até a formação das imagens, foi possível conhecer as fontes incertezas envolvidas e estimar a incerteza de medição da técnica.
\end{abstract}

Palavras-chave: Ensaios não destrutivos; Ultrassom; Tempo de Voo da Onda Difratada - ToFD; Metrologia.

\section{Time of Flight Diffraction Technique (ToFD) Assists in Quality Assurance of Parts Production and Mechanical Structures}

\begin{abstract}
The Time of Flight Diffraction Technique (ToFD) is a technique of non-destructive testing by ultrasound used for detecting faults and discontinuities in different components, ensuring the quality, reliability and safety. It is widely used in industry, particularly in the evaluation of mechanical parts and structures. It is widely used in the sectors oil/petrochemical, chemical, aeronautical, aerospace, steel, shipbuilding, electromechanical, pulp and paper, among others. The development and implementation of this technique was based on ISO 16828:2012 (Non-Destructive testing - Ultrasonic Testing - Time-of-Flight Diffraction Technique as a Method for Detection and Sizing of Discontinuities). Controlling the different characteristics of the system, from the specification of the ultrasonic characteristics, to the imaging technique, it was possible to identify the sources of uncertainty and estimate the ToFD measurement uncertainty.
\end{abstract}

Key-words: Non destructive testing; Ultrasound; Time of Flight Diffraction Technique - ToFD; Metrology.

\section{Introdução}

Os ensaios não destrutivos (END) são amplamente utilizados na indústria, principalmente na avaliação de peças e estruturas mecânicas para a identificação de defeitos de fabricação, corrosão, falhas devido a tensões ou imperfeições em soldas. Os END constituem uma das principais ferramentas do controle da qualidade de materiais e produtos, contribuindo para garantir a qualidade dos bens e serviços, reduzir os custos, preservar a vida e o meio ambiente. Sendo assim, são um fator de competitividade para as empresas que os utilizam. São largamente utilizados nos setores petróleo/petroquímico, químico, aeronáutico, aeroespacial, siderúrgico, naval, eletromecânico, papel e celulose, entre outros. Dentre as principais técnicas de END, as mais utilizadas são baseadas no uso do ultrassom, como, por exemplo, para medição de espessura ou localização de fendas e descontinuidades no material sob ensaio. Uma destas técnicas é a Técnica Tempo de Voo da Onda Difratada (Time of Flight Diffraction - ToFD), que permite a formação de imagens do objeto ensaiado, possibilitando uma avaliação visual (Associação Brasileira de Ensaios Não Destrutivos, 2013).

A técnica ToFD foi implementada no Laboratório de Ultrassom do Inmetro (Labus) com base na norma ISO 16828:2012 (Non-Destructive testing - Ultrasonic Testing - Time-of-Flight Diffraction Technique as a Method for Detection and Sizing of Discontinuities) (International Organization for Standardization, 2012a), permitindo que se tenha controle sobre as diferentes características do sistema, desde a especificação das suas características ultrassônicas, da formação de imagens e das fontes de 
incerteza envolvidas. Além disso, a técnica ToFD foi comparada a outra técnica de END, para mostrar a importância da escolha adequada de uma técnica devido as características do ensaio.

\section{Técnica Tempo de Voo da Onda Difratada - ToFD}

O ToFD é uma técnica de END por ultrassom que vem sendo aplicada com sucesso para o dimensionamento de defeitos em seções transversais de peças e equipamentos. Foi inventado no Reino Unido na década de 1970, inicialmente, como uma ferramenta de pesquisa. No entanto, o método foi inicialmente ignorado pelos especialistas. Finalmente em 1996, um artigo científico europeu foi anunciado, e graças a esta iniciativa e a alguns trabalhos publicados, o ToFD começou a ser utilizado como uma das principais técnicas de END por ultrassom (Hecht, 1997).

Devido à amplitude do sinal refletido não ser o melhor método para o dimensionamento de defeitos, uma vez que a amplitude de um sinal ultrassônico retroespalhado depende fortemente da orientação e características das descontinuidades, o ToFD utiliza o tempo de voo de um pulso de ultrassom para determinar a posição de um refletor (Baby, 2002). A técnica baseia-se no uso de dois transdutores angulares colocados paralelamente sobre a mesma superfície, assim como demonstrado na Figura 1a, com o objetivo de que um pulso ultrassônico gerado por um transdutor (Emissor) seja recebido pelo transdutor oposto (Receptor) (Silk e Lidington, 1975).

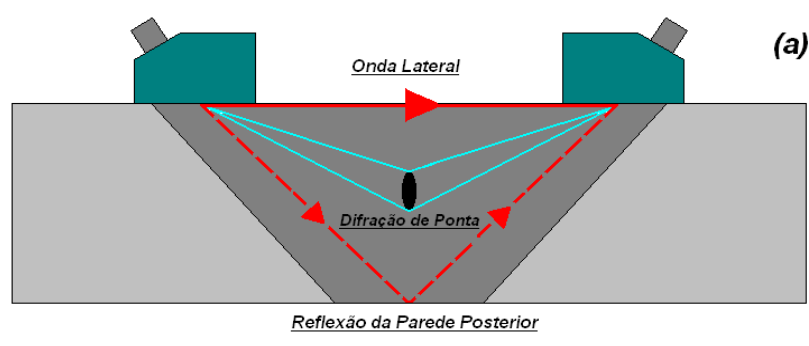

(b)

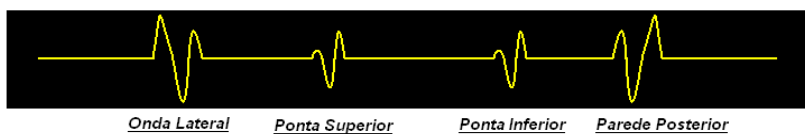

Figura 1. (a) Formação de ondas características do ToFD (b) Exemplo de um sinal tipo A-scan característico do ToFD (adaptado de Mayworm et al., 2016).

Os sinais gerados pelo transdutor emissor formam duas ondas, uma que viaja ao longo da superfície e outra que é a refletida na parede inferior. Quando um feixe ultrassônico incide em uma descontinuidade, além dos fenômenos já conhecidos de reflexão e transmissão, ocorre também à difração sônica, que consiste na geração de uma onda circular que se propaga em todas as direções. A deteç̧ão das ondas difratadas torna possível estabelecer a presença de descontinuidades (Silk e Lidington, 1975). Medindo-se o tempo de voo do pulso, a profundidade de uma extremidade da descontinuidade pode ser calculada por trigonometria, podendo-se então dimensioná-la. A dimensão da descontinuidade é sempre determinada a partir do tempo de voo das ondas difratadas. Na Figura $1 \mathrm{~b}$, pode-se observar a forma de sinal recebido (A-Scan) quando detectado algum tipo de descontinuidade (Baby, 2002)

O ToFD gerar uma imagem do tipo D-scan a partir de um processamento dos sinais A-scan. O mostrador tipo A ( $A$-scan display) é basicamente um gráfico de amplitude (representada no eixo vertical) versus tempo de voo (representado no horizontal) (Subbaratnam et al., 2011). Conhecendo-se a velocidade de propagação do pulso ultrassônico no meio é possível converter o tempo de voo do sinal em distância. Ao organizar as formas de onda A-scan lado a lado, em correspondência com cada ponto de varredura, pode-se gerar uma imagem D-scan característica do ToFD (Sinclair, 2010). Assim, o mostrador tipo D ( $D$-scan display) apresenta uma imagem em escala de cinza, gerada a partir dos sinais A-scan não retificados, representando uma vista da seção transversal perpendicular ao objeto ensaiado (Subbaratnam et al., 2011). Por convenção, os sinais positivos tendem para a cor branca, enquanto que os sinais negativos para a cor preta, onde a amplitude se torna a escala de cores como demonstrado na Figura 2 (International Organization for Standardization, 2012a). 

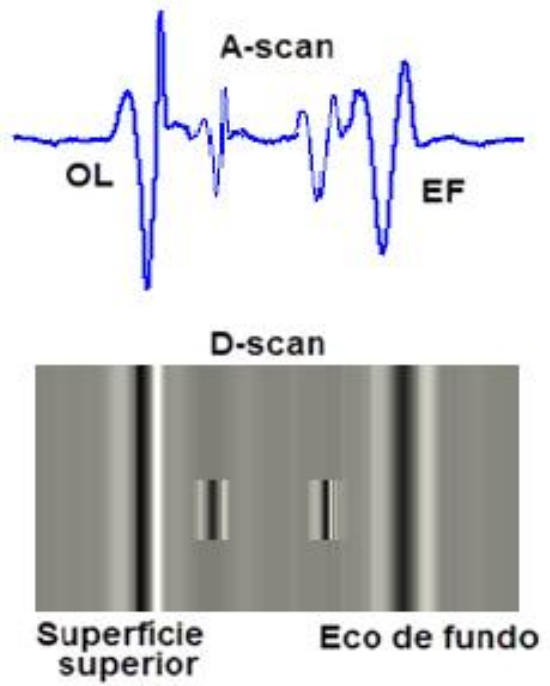

Figura 2. Sinal A-Scan característico da técnica ToFD e a imagem D-Scan gerada a partir do sinal A-Scan (adaptado de Mayworm et al., 2016).

\section{Materiais e Métodos}

A fim de que a técnica ToFD possa ser comparada a outra técnica de END e para mostrar a importância da escolha adequada de uma técnica devido as características do ensaio, utilizou-se também a técnica pulso-eco. O objetivo é que ao final dos ensaios possa haver uma comparação entre os resultados. Para tal, desenvolveu-se um aplicativo que permite gerar imagens a partir dos sinais captados por meio de um sistema de aquisição, tanto da técnica ToFD, quanto da técnica pulso-eco. Os ensaios foram feitos em um mesmo bloco padrão que possui descontinuidades conhecidas. Mais detalhes são apresentados a seguir.

\subsection{Corpo de prova}

Para a realização dos ensaios foi utilizado um bloco de referência para ultrassom do tipo 1 (International Organization for Standardization, 2012b). O bloco foi utilizado tanto para o ensaio da técnica ToFD quanto para técnica de pulso-eco, a fim de comparar os resultados de ambos ensaios. O bloco padrão utilizado possui várias descontinuidades com dimensões conhecidas, as quais foram calibradas no Laboratório de Metrologia Dimensional do Inmetro (Lamed). Apesar do bloco possuir suas dimensões calibradas, foi necessária a realização da calibração da sua velocidade ultrassônica, já que é um parâmetro de entrada no aplicativo desenvolvido e é utilizada para a conversão do tempo de voo em distância.

\subsection{Sistema de aquisição de sinais}

Na montagem do sistema de aquisição de sinais foram utilizados os seguintes equipamentos: um detector de falhas modelo EPOCH 600, da marca Olympus, como gerador de pulsos; um osciloscópio modelo DSO 3024A, da marca Agilent Technologies; dois transdutores (emissor e receptor) modelo C543-SM, frequência de $5 \mathrm{MHz}$ e diâmetro de 6 mm, da marca Olympus (tanto para o ensaio pulso-eco, quanto para o ToFD); duas sapatas angulares de 60 de acrílico, modelo ABSA-4T-60, da marca Olympus; Atenuador modelo 837, da marca KAY Elemetrics Corp.; um cabo de comunicação USB, mel como elemento acoplante e um computador contendo o aplicativo desenvolvido em Labview (descrito em detalhes no item 3.3). Na Figura 3 é apresentada a montagem do sistema de aquisição, onde podem ser vistos os instrumentos utilizados. 


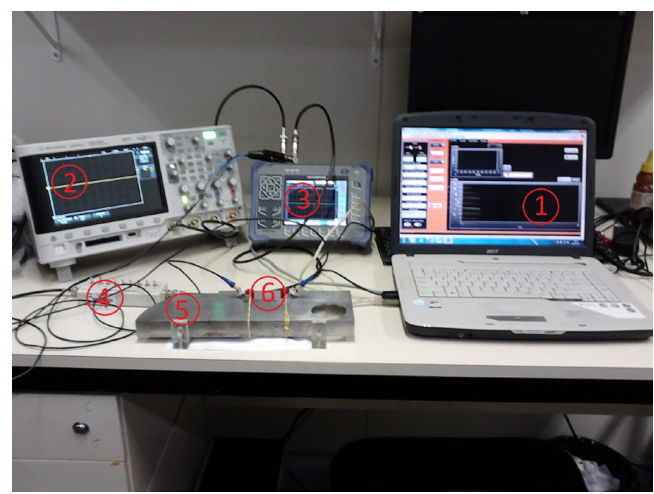

Figura 3. Montagem do sistema de aquisição de sinais: 1) Computador contendo o aplicativo desenvolvido em Labview; 2) osciloscópio DSO 3024A; 3) detector de falhas EPOCH 600; 4) atenuador 837; 5) bloco padrão; 6) transdutores e sapatas da Olympus.

\subsection{Aplicativo}

O aplicativo tem como função gerar as imagens construídas através dos sinais captados pelo sistema de aquisição, sendo muito comum a sua utilização em ensaios não destrutivos, pois são capazes de apresentar resultados confiáveis, além de serem mais práticos e na maioria das vezes, aumentando a velocidade e a confiabilidade da inspeção (National Instruments, 2013).

Optou-se por utilizar o Labview ${ }^{\mathrm{TM}}$ (National Instruments, USA) para o desenvolvimento do aplicativo, pois é um software construído especificamente para tarefas executadas por engenheiros e cientistas, composto por ferramentas capazes de criar e implantar sistemas de medição e controle. Complementarmente, o Labus possui experiência no desenvolvimento de sistemas usando o Labview ${ }^{\mathrm{TM}}$. No aplicativo desenvolvido é possível utilizar dois diferentes tipos de técnicas: ToFD e Pulso-Eco. Na Figura 4, é apresentado o painel frontal do aplicativo desenvolvido.

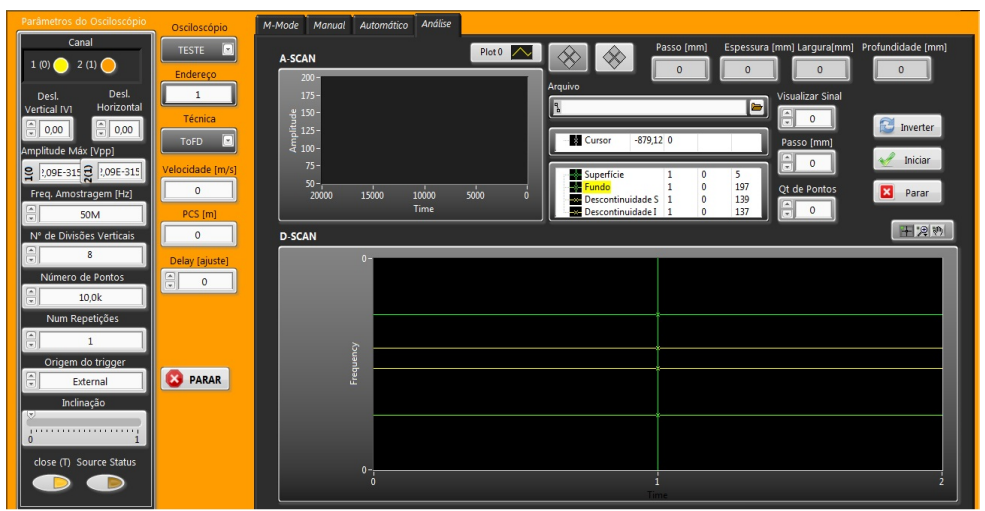

Figura 4. Painel frontal do aplicativo desenvolvido em Labview.

Para a medição da espessura do objeto sob ensaio e das possíveis descontinuidades nele presentes, o aplicativo possui dois pares de cursores que calculam a espessura e as descontinuidades a partir do tempo de voo obtido dos sinais adquiridos. A Equação 1, Equação 2 e Equação 3 são utilizadas para os cálculos das dimensões no ToFD (Mondal e Sattar, 2000).

$H=S^{2}-\frac{c^{2} \cdot t^{2}}{4}$

$L=\frac{1}{2} \sqrt{c^{2} \cdot t^{2}-S^{2}}$

$D=H-\frac{1}{2} \sqrt{c^{2} \cdot t^{2}-S^{2}}$

No qual $S$ é a distância de separação entre os transdutores (Probe Center Separation - PCS) [m], $L$ é o comprimento da descontinuidade $[\mathrm{m}], D$ é a profundidade da descontinuidade $[\mathrm{m}], c$ é a velocidade de propagação ultrassônica no aço [m/s], $t$ é o tempo de voo do sinal [s] e $\mathrm{H}$ a espessura do material [m]. 


\subsection{Determinação da velocidade ultrassônica de propagação dos blocos}

A calibração das velocidades transversal e longitudinal do bloco de referência foi realizada utilizando-se um transdutor de ondas transversais, modelo V155-RB, da marca Olympus, um transdutor da marca Olympus, modelo C543-SM, um osciloscópio da marca Agilent Technologies, modelo DSO 3024A e um paquímetro.

O procedimento usado na calibração da velocidade faz parte dos serviços prestados pelo Labus e é baseado na norma ISO 2400 Non-destructive testing - Ultrasonic testing - Specification for calibration block No. 1 (International Organization for Standardization, 2012b). Portanto, o processo se iniciou com a marcação de quatro diferentes pontos no bloco de referência. Nos quatro pontos foram medidas as suas respectivas espessuras, sendo este processo repetido três vezes. Logo, para cada ponto obteve-se três valores de espessura. O mesmo processo foi realizado para a medição do tempo de voo do pulso ultrassônico, utilizando-se os mesmos pontos e repetindo a medição do tempo de voo, por meio do osciloscópio, três vezes em cada um dos 4 pontos. Determinou-se, então, a média da espessura e do tempo de voo em cada ponto, e a partir destas, calculou-se a velocidade para cada ponto de medição. Como esse processo foi realizado em quatro diferentes pontos do bloco, calcularam-se quatro valores de espessura e tempo, dos quais se calculou a média para a obtenção do valor final médio da velocidade do bloco. Cabe ressaltar que o mesmo procedimento foi utilizado para a determinação tanto da velocidade longitudinal quanto da velocidade transversal.

\subsection{Cálculo das incertezas de medição}

Com o intuito de apresentar uma indicação quantitativa da qualidade dos resultados, realizou-se a estimativa da incerteza de medição.

Para uma relação genérica $h=f\left(x_{j}\right)$, a fórmula geral para o modelo de incerteza é apresentado na Equação 4 (Bureau International des Poids et Mesures, 2008).

$u_{c}^{2}=\sum_{j=l}^{N}\left(\frac{\partial h}{\partial x_{j}}\right)^{2} \cdot u_{j}^{2}$

Onde $u_{c}$ é a incerteza padrão combinada associada com o resultado final da medição (ou cálculo) de $h$, e $u_{j}$ a incerteza padrão, avaliada como tipo A ou tipo B, associada a cada parâmetro variável $x_{j}$ utilizado para expressar o valor de $h$.

Na grande maioria dos casos, a média aritmética é a melhor estimativa para o valor esperado de uma quantidade que varia aleatoriamente e para a qual se tem $n$ leituras independentes obtidas em condições de repetibilidade. Assim, quando a estimativa de uma grandeza de entrada foi obtida de $n$ medições em condições de repetitividade, a incerteza do tipo A é obtida pela estimativa do desvio-padrão da média, dada pela Equação 5 (Bureau International des Poids et Mesures, 2008).

$u_{A}=\frac{s}{\sqrt{n}}$

Na qual:

$u_{a}=$ incerteza do Tipo A;

$s=$ desvio-padrão;

$n=$ número de medições realizadas.

Para uma estimativa de uma grandeza de entrada $x_{i}$, que não tenha sido obtida de observações repetidas, a incerteza padrão $u_{b}\left(x_{i}\right)$ é avaliada pelo julgamento específico baseado em todas as informações disponíveis na variabilidade de $x_{i}$. No conjunto destas informações são incluídos: informações prévias de medição, experiência ou conhecimento geral do comportamento e propriedades dos instrumentos e materiais relevantes, especificações do fabricante, informações de relatórios de calibração e outras especificações e a incerteza transmitida pelas informações de referências obtidas de manuais (Bureau International des Poids et Mesures, 2008).

\subsection{Incerteza de medição das velocidades de propagação do bloco}

Para o cálculo da incerteza de medição da velocidade de propagação do bloco, tanto transversal quanto longitudinal, foi necessário o cálculo das incertezas combinadas do tempo de voo e da espessura (distância) utilizados para o cálculo da velocidade.

Utilizou-se, então, o desvio padrão da média final obtida das quatro médias (pontos 1 a 4 do bloco) de espessura do bloco e foram determinadas as incertezas do tipo A e tipo B, a fim de se calcular a incerteza combinada. A incerteza do tipo A, foi estimada a partir do desvio padrão das quatro médias (conforme Equação 5) e a incerteza do tipo $B$, proveniente da resolução do paquímetro digital $(0,01 \mathrm{~mm})$ dividida por $2 \sqrt{3}$, por ser considerada uma distribuição retangular.

O mesmo aconteceu para o cálculo da incerteza de medição do tempo de voo, onde se utilizou o desvio padrão das quatro médias (pontos 1 a 4) para o cálculo da incerteza do tipo A, e a incerteza do tipo B foi calculada em função da resolução do 
equipamento utilizado na medição do tempo, neste caso o osciloscópio. De acordo com o manual do osciloscópio a sua incerteza do tipo B pode ser estimada de acordo com a Equação 6.

$u_{B}^{t}=25$ ppm.t

No qual ppm representa partes por milhão.

Em seguida, foram obteve-se as incertezas combinadas do tempo e da espessura, podendo-se então calcular a incerteza combinada final das velocidades longitudinal e transversal, por meio da Equação 7 e Equação 8, respectivamente.

$u_{c f}^{v l}=\sqrt{\left(u_{c}^{d} \cdot \frac{\partial v}{\partial d}\right)^{2}+\left(u_{c}^{t l} \cdot \frac{\partial v}{\partial t}\right)^{2}} \therefore u_{c f}^{v l}=\sqrt{\left(u_{c}^{d} \cdot \frac{2}{t}\right)^{2}+\left(u_{c}^{t l} \cdot \frac{2 \cdot d}{t^{2}}\right)^{2}}$

$u_{c f}^{v t}=\sqrt{\left(u_{c}^{d} \cdot \frac{\partial v}{\partial d}\right)^{2}+\left(u_{c}^{t t} \cdot \frac{\partial v}{\partial t}\right)^{2}} \therefore u_{c f}^{v t}=\sqrt{\left(u_{c}^{d} \cdot \frac{2}{t}\right)^{2}+\left(u_{c}^{t t} \cdot \frac{2 \cdot d}{t^{2}}\right)^{2}}$

No qual: $u_{c f}^{v l}$ é a incerteza combinada final da velocidade longitudinal [mm/s], $u_{c}^{d}$ é a incerteza combinada da distância (espessura do bloco) [mm], $u_{c}^{t l}$ é a incerteza combinada do tempo longitudinal [s], $u_{c f}^{v t}$ é a incerteza combinada final da velocidade transversal [mm/s], $u_{c}^{t t}$ é a incerteza combinada do tempo transversal [s].

Por fim, determinou-se incerteza expandida $\bigcup_{\mathrm{EXP}}$, demonstrada na Equação 9, para uma probabilidade de 95,45\% e fator de abrangência $\mathrm{k}=2$.

$U_{E X P}=k \cdot u_{c}$

\subsection{Incerteza de medição dos cursores}

As incertezas de medição foram calculadas a partir das equações utilizadas nos cursores do programa, tanto para a técnica ToFD quanto para a técnica pulso-eco. Para o cálculo da incerteza de medição dos cursores utilizou-se apenas as incertezas do tipo B. Como as variáveis das equações utilizadas para o cálculo dos cursores são $S$ (PCS), c (velocidade) e $t$ (tempo) utilizou-se no caso de $c$ e $t$ as incertezas do tipo B determinadas para o cálculo da incerteza do bloco. Logo, foi necessário calcular apenas a incerteza do tipo B para a régua (utilizada para medir PCS), que se trata de uma distribuição triangular com resolução de 1 mm. Após os cálculos de todas essas incertezas calculou-se a incerteza combinada final para cada medição de $E$ (espessura pulso-eco), $H$ (espessura ToFD), $D$ (profundidade ToFD) com os cursores, nos quais podem ser observadas na Equação 10 , Equação 11 e Equação 12. Ao final calculou-se a incerteza expandida $(k=2)$.

$$
\begin{aligned}
& u_{c f}^{E}=\sqrt{\left(u_{c f}^{v l} \cdot \frac{\partial d}{\partial v}\right)^{2}+\left(u_{c f}^{t l} \cdot \frac{\partial d}{\partial t}\right)^{2}} \\
& u_{c f}^{H}=\sqrt{\left(u_{B}^{S} \cdot(2 \cdot S)\right)^{2}+\left(u_{B}^{c t} \cdot\left(\frac{c \cdot t^{2}}{2}\right)\right)^{2}+\left(u_{B}^{t t} \cdot\left(\frac{c^{2} \cdot t}{2}\right)\right)^{2}}
\end{aligned}
$$

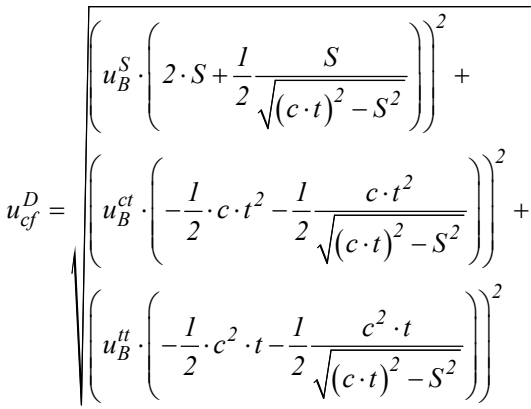

No qual: $u_{c f}^{E}$ é a incerteza combinada final do par de cursores espessura (Pulso-Eco) [mm], $u_{c f}^{v l}=u_{c f}^{c l}$ é a incerteza combinada final da velocidade longitudinal $[\mathrm{mm} / \mathrm{s}], u_{c f}^{t l}$ é a incerteza combinada final do tempo longitudinal [s], $u_{c f}^{D}$ é a incerteza combinada final do par de cursores profundidade (ToFD) [mm], $u_{c f}^{H}$ é a incerteza combinada final do par de cursores espessura (ToFD) [mm], $u_{B}^{S}$ é a incerteza do tipo B do PCS [mm], $u_{B}^{v t}=u_{B}^{c t}$ é a incerteza do tipo B da velocidade transversal [mm/s], $u_{B}^{t t}$ é a incerteza do tipo $B$ do tempo transversal $[\mathrm{s}]$. 


\section{Resultados e Discussão}

A seguir são apresentados os resultados obtidos na calibração do bloco e por meio do aplicativo, tanto para a técnica ToFD quanto para a técnica pulso-eco.

\subsection{Calibração da velocidade ultrassônica do bloco padrão}

Os valores médios de espessura do bloco e dos tempos de voo longitudinal e transversal, bem como seus respectivos desvios-padrão, obtidos a partir das medições em quatro diferentes posições do bloco sob ensaio são apresentados na Tabela 1. Os valores médios de velocidade longitudinal e transversal calculados para todo o bloco, bem como suas respectivas incertezas expandidas, são apresentados na Tabela 2.

Tabela 1. Valores médios de espessura do bloco e dos tempos de voo longitudinal e transversal, bem como seus respectivos desvios-padrão, obtidos a partir das medições em quatro diferentes posições do bloco sob ensaio.

\begin{tabular}{ccc}
\hline & Média & Desvio padrão \\
Espessura média $[\mathrm{mm}]$ & 25,04 & 0,03 \\
Tempo de voo médio da onda longitudinal $[\mu \mathrm{s}]$ & 8,50 & 0,01 \\
Tempo de voo médio da onda transversal $[\mu \mathrm{s}]$ & 15,20 & 0,01 \\
\hline
\end{tabular}

Tabela 2. Valores médios de velocidade longitudinal e transversal, bem como suas respectivas incertezas expandidas.

\begin{tabular}{llc}
\hline & Média & Incerteza expandida \\
Velocidade Longitudinal $[\mathrm{m} / \mathrm{s}]$ & 5891,8 & 9,8 \\
Velocidade Transversal $[\mathrm{m} / \mathrm{s}]$ & 3294,7 & 4,6 \\
\hline
\end{tabular}

\subsection{Ensaio do bloco padrão}

Os ensaios aqui realizados basearam-se no método de aquisição manual, adquirindo somente um ponto, em diferentes regiões do bloco e comparando-as. Para analisar o comportamento das técnicas ToFD e pulso-eco, foram escolhidas duas regiões do bloco. Uma região sem falhas, no qual pode ser observada na região 1 da Figura 5, e outra contendo rasgos de 4 mm de profundidade nas faces superior e inferior do bloco, no qual pode ser observada na região 2 da Figura 5.

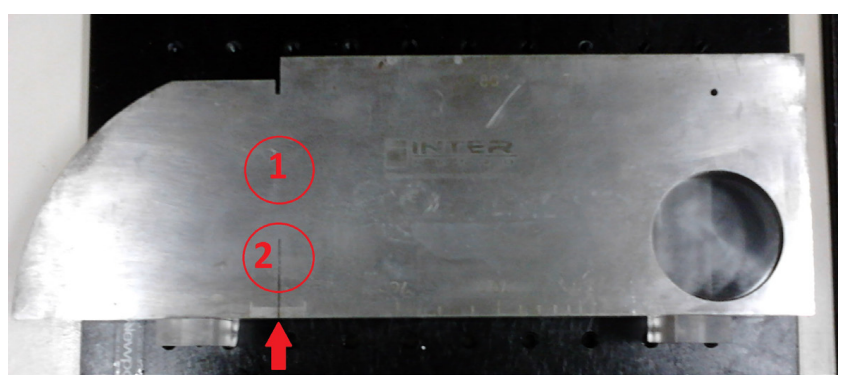

Figura 5. Pontos onde foram realizados os ensaios. Ponto 1: região sem falhas, Ponto 2: região com rasgos de $4 \mathrm{~mm}$ nas partes inferior e superior.

De acordo com a norma ISO 16828:2012 (Non-Destructive testing - Ultrasonic Testing - Time-of-Flight Diffraction Technique as a Method for Detection and Sizing of Discontinuities), para espessuras de até $50 \mathrm{~mm}$, utiliza-se um ajuste inicial para direcionar o feixe a $2 / 3$ da espessura, quando não se tem ideia da localização do defeito da amostra (ISO 16828; 2012) (International Organization for Standardization, 2012a; Associação Brasileira de Normas Técnicas, 2013).

Por meio dessa conversão, calculou-se o PCS necessário, sendo este igual a $57 \mathrm{~mm}$.

Na Figura 6, tem-se a imagem gerada a partir dos sinais adquiridos nas regiões 1 e 2 pela técnica ToFD. Podem-se observar dois sinais principais, sendo eles o da onda lateral (Figura 6 - Ponto 1) e o da reflexão do fundo (Figura 6 - Ponto 2), observa-se ainda outra linha na parte referente ao rasgo inferior (Figura 6 - Ponto 3), porém não foi possível identificar o rasgo superior, localizado próximo à superfície. Outro fator interessante é o desaparecimento da onda lateral na área referente aos rasgos, que acontece devido ao rasgo superior impedir que a onda lateral se propague sobre a superfície do material, esse efeito é uma indicação clara de uma descontinuidade ou trinca superficial. 


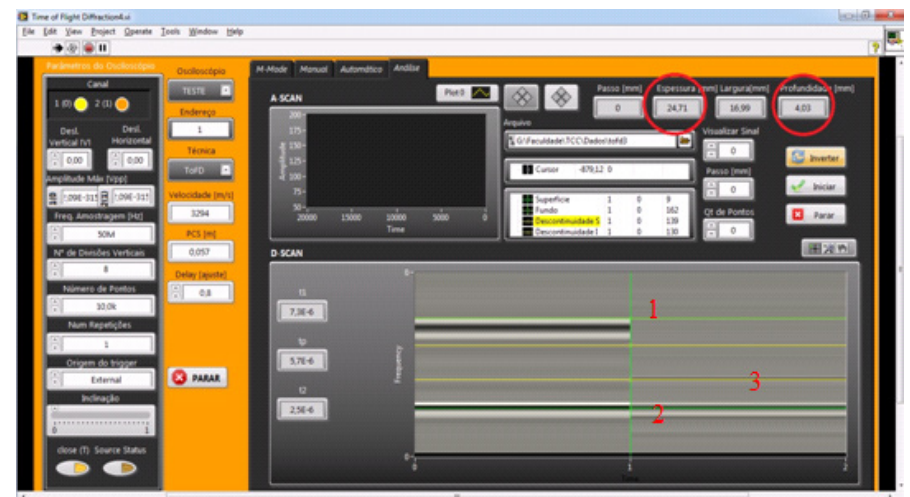

Figura 6. Medição da espessura e das descontinuidades realizada utilizando-se o programa em Labview para a técnica ToFD.

Após a identificação das principais ondas (possíveis descontinuidades) os cursores foram utilizados para dimensionar as descontinuidades. Os valores medidos com os cursores, assim como suas respectivas incertezas, são apresentados na Tabela 3.

Pode-se observar que a espessura calculada pela técnica ToFD é próxima a calibrada dimensionalmente, porém não tanto quanto a calculada através da técnica pulso-eco (Tabela 3). Por meio dos cursores foi possível também medir a descontinuidade inferior, onde se obteve o valor de 4,03 mm (Tabela 3). O cálculo das respectivas incertezas é apresentado, a seguir, no item 5.3 .

A fim de verificar se o valor obtido na medição realizada pela técnica ToFD pode ser considerado estatisticamente não diferente a dimensão da descontinuidade, é aplicado o teste de diferença entre médias. Considera-se a hipótese nula de igualdade entre médias e a hipótese alternativa de diferença entre médias, sendo a estatística teste $t_{\text {calc }}$ dada pela Equação 13 .

$t_{\text {calc. }}=\frac{X-\mu}{\frac{u_{c f}}{\sqrt{n}}}$

Onde $X$ é o valor médio das medições de cada técnico, $u_{c f}$ sua respectiva incerteza combinada final e $n$ o número de medições.

O valor obtido para $t_{\text {calc }}$ é comparado com o de $t_{\text {tab }}$ extraído da tabela da distribuição $t$ de Student, em função dos graus

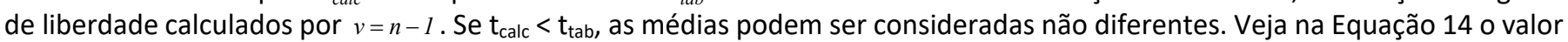
de $t_{\text {calc }}$.

$t_{\text {calc. }}=\frac{4,03-4}{\frac{0,045}{\sqrt{3}}} \therefore t_{\text {calc. }}=\frac{4,03-4}{\frac{0,045}{\sqrt{3}}} \therefore t_{\text {calc. }}=1.154$

Temos, para $n=3$ e um nível de confiança de $95 \%, t_{\text {tab }}=4,303$, portanto, podemos dizer que os valores são estaticamente não diferentes.

Tabela 3. Comparação entre os resultados medidos usando-se as técnicas ToFD e pulso-eco e os valores oriundos da calibração dimensional do bloco padrão no Lamed/Inmetro.

\begin{tabular}{ccc}
\hline & Medida & Incerteza expandida \\
Espessura calibrada $[\mathrm{mm}]^{1}$ & 25,000 & 0,003 \\
Espessura medida pela técnica Pulso-eco $[\mathrm{mm}]$ & 25,130 & 0,042 \\
Espessura medida pela técnica ToFD [mm] & 24,710 & 0,093 \\
Profundidade definida em norma $[\mathrm{mm}]^{2}$ & 4,00 & - \\
Profundidade medida pela técnica Pulso-eco $[\mathrm{mm}]$ & 4,100 & 0,042 \\
Profundidade medida pela técnica ToFD [mm] & 4,03 & 0,50 \\
\hline
\end{tabular}

${ }^{1}$ Valor retirado do certificado de calibração; ${ }^{2}$ Valor retirado da Norma ISO 2400 (International Organization for Standardization , 2012b) .

\section{Conclusão}

A técnica de ultrassom pulso-eco não se mostrou eficiente na detecção dos rasgos do bloco, mostrando-se ineficiente para descontinuidades perpendiculares em relação à superfície de ensaio. Porém, a técnica se mostrou eficiente para o dimensionamento, tanto da espessura quanto da profundidade do rasgo, com erros de $0,52 \%$ e $2,5 \%$, respectivamente. O ToFD, por sua vez, mostrou-se sensível aos rasgos do bloco e a presença da descontinuidade, além de apresentar resultados consistentes com os valores calibrados. Para o dimensionamento do rasgo, a técnica ToFD teve erro de 0,75\%, quase 4 vezes 
menor do que o erro da técnica pulso-eco. Todavia, o ToFD não se mostrou tão eficiente como a técnica pulso-eco para o dimensionamento da espessura do bloco, com erro de $0,84 \%$ contra os 0,52\% da técnica Pulso-Eco.

Considerando os resultados obtidos, conclui-se que o ToFD é uma alternativa adequada para a detecção de falhas e descontinuidades, independente da sua orientação e dimensão. Porém, fica clara a necessidade do uso adequado das diferentes técnicas de END por ultrassom para a detecção de falhas e descontinuidades, bem como do estudo das fontes de incerteza que podem influenciar os resultados. Caso estes fatores não sejam considerados, os resultados poderão ser negativamente influenciados, comprometendo a confiabilidade da inspeção.

\section{Agradecimentos}

Os autores agradecem ao CNPq e a Faperj pelo apoio financeiro.

\section{Referências}

Associação Brasileira de Ensaios Não Destrutivos - ABENDI. [página da internet]. São Paulo: ABENDE; 2013 [acesso em 24 jul. 2013 ]. Disponível em: www.abende.org.br

Associação Brasileira de Normas Técnicas - ABNT. ABNT NBR 16196:2013 - Ensaios não destrutivos - Ultrassom - Uso da técnica de tempo de percurso da onda difratada (ToFD) para ensaio em soldas. Rio de Janeiro: ABNT; 2013.

Baby S. Time of Flight Diffraction (ToFD) technique for accurate sizing of surface breaking cracks. OR Insight, 2002;45(6):426-430. http://dx.doi.org/10.1784/insi.45.6.426.52885.

Bureau International des Poids et Mesures - BIPM. JCGM 100:2008 - Evaluation of measurement data - Guide to the expression of uncertainty in measurement. France: BIPM; 2008.

Hecht A. Time of Flight Diffraction Technique (ToFD) - an ultrasonic testing a method for all applications? NDT Journal. 1997;2(9).

International Organization for Standardization - ISO. ISO 16828:2012 - Non-destructive testing. Ultrasonic testing. Time-of-flight diffraction technique as a method for detection and sizing of discontinuities. Genebra: ISO; 2012a.

International Organization for Standardization - ISO. ISO 2400:2012 - Non-destructive testing. Ultrasonic testing. Specification for calibration block. No. 1. Genebra: ISO; 2012b.

Mayworm RC, Costa-Félix RPB, Alvarenga AV. Aspectos metrológicos da Técnica Tempo de Voo da Onda Difratada (ToFD) para Utilização em Ensaios Não Destrutivos (ENDs) por Ultrassom. Soldagem e Inspeção. 2016;21(4):406-416. http://dx.doi.org/10.1590/01049224/si2104.02.

Mondal S, Sattar TP. An overview TOFD method and its Mathematical Model. e-Journal of NDT. 2000;5(4).

National Instruments. O que é o LabVIEW?. São Paulo: National Instruments; 2013. [acesso em 29 maio 2013. Disponível em: www.ni.com/labview/

Silk MG, Lidington BH. The potential of scattered of diffracted ultrasound in the determination of crack depth. Non-Destructive Testing. 1975;8(3):146-151.

Sinclair AN. Enhancement of ultrasonic images for sizing of defects by time-of-flight diffraction. NDT \& E International. 2010;43(3):258-264.

Subbaratnam R, Saju T, Abraham B, Venkatraman B, Baldev R. Immersion and ToFD (I-TOFD): a novel combination. J Nondestruct Eval. 2011;30(3):137-142. http://dx.doi.org/10.1007/s10921-011-0101-0. 